\title{
THE DELTA SCUTI STAR GX PEGASI: A THEORETICAL INVESTIGATION OF ITS POWER SPECTRUM
}

\author{
E. MICHEL, M. J. GOUPIL, Y. LEBRETON and A. BAGLIN \\ Observatorie de Paris, DASGAL, URA CNRS 335, France
}

\section{Introduction}

Target of a STEPHI multisite campaign, the Delta Scuti star GX Pegasi has been found to oscillate with at least five simultaneous, close frequencies (table I).

TABLE I

GX Peg's frequencies $( \pm 0.25 \mu \mathrm{Hz})$ (from Michel et al., 1992a)

\begin{tabular}{cccccc}
\hline & $\nu_{1}$ & $\nu_{2}$ & $\nu_{3}$ & $\nu_{4}$ & $\nu_{5}$ \\
\hline frequency $(\mu \mathrm{Hz})$ & 187.2 & 227.6 & 230.4 & 237.5 & 240.5 \\
\hline
\end{tabular}

Mode identification together with informations about the star that such an identification can provide are outlined below (see also Michel et al, 1992b).

The mode identification is carried out by means of a comparison between the observed frequencies and the adiabatic frequencies of models appropriate to this star. Models that match GX Peg's position in a Hertzsprung-Russell diagram have masses in the range $1.9-2 M_{\odot}$. When included, convective core overshoot is handled as in Maeder and Meynet (1989). According to these models, GX Peg is a rather evolved, main sequence star.

Adiabatic frequencies, $\nu_{n l}$ (degree $l$, radial order $n$ ), and eigenfunctions are calculated with a programme kindly supplied by Christensen-Dalsgaard (1982).

\section{Mode Identification}

On the basis of geometrical visibility effects (Christensen-Dalsgaard et al., 1982) as well as energy and mode trapping arguments (Lee 1985, Dziembowski and Krolikowska, 1990), we consider modes $l=0, l=1$ as most probably detected.

Only the first and second radial overtones belong to the observed frequency interval, in the range of effective temperatures and masses of interest. The frequency $\nu_{1}$ is identified with the first radial overtone. Comparison 
between theoretical and observed period ratios shows that $\nu_{3}$ corresponds to the second radial overtone, when Livermore opacities (Rogers and Iglesias, 1991) are used.

Models without overshoot offer the possible choice of $l=1$ modes with radial order $n=2$ or 3 for the three other observed frequencies. As a consequence of their different evolutionary status, models with convective core overshoot (which has to exist to some extent, see also Dziembowski et al., 1991) only allow the mode $l=1, n=3$, whose frequency then falls very close to the observed frequency, $\nu_{4}$.

We therefore retain the following identification as the most probable one: $\nu_{1}, \nu_{2}$ as the radial first and second overtones modes and $\left(\nu_{3}, \nu_{4}, \nu_{5}\right)$ as the triplet $l=1, n=3$ with azimuthal orders $m=1,0,-1$ respectively.

\section{First Step Towards Seismology of GX Pegasi}

The $l=1$ modes in the frequency range $\nu_{1}-\nu_{5}$ all have amplitude in the inner gravity propagative region except the $(n=3)$ mode which is trapped in the envelope. It is precisely this last mode which is detected in GX Peg's pulsation. This supports the idea that mode trapping plays a role mode selection as suggested by Dziembowski et al. (1991).

Assuming that the star (which belongs to a spectroscopic binary system) is synchronized $\left(\Omega_{\text {rot }}=5 \mu \mathrm{Hz}\right)$, the value of the splitting $\left(\nu_{5}-\nu_{2}\right) / 2$ shows that GX Peg's rotation cannot be solid. If the surface rotation is $\Omega_{\text {rot }}$ and a linear variation of the rotation with radius is assumed, the core rotation then amounts up to $\sim 2 \Omega_{\text {rot }}$.

Departure from equidistance for the triplet $l=1$ (expectantly due to rapid rotation and binarity) is found very asymmetric and large but in agreement with theoretical calculations (Saio, 1981; Dziembowski et al., 1990).

The quantity $C_{n l}=\left(\nu_{n l}-\nu_{n 0}\right) /\left(\nu_{n 0}-\nu_{n-1,0}\right)$ sharply varies with $1 / \nu_{n l}$ when the mode $(n, l)$ develops amplitude in the inner gravity propagative region. This variation is only slightly sensitive to mass and to external regions but very sensitive to core overshoot. $C_{n l}$ then tests the deep regions of convective core models. Its variation is found very close, indeed, to the asymptotic arctangent behavior expected for mixed modes, which depends on the extension of the inner evanescent and gravity propagative regions.

The observed value $C_{31}$ for GX Peg falls slightly below the theoretical $C_{31}$ curves. The discrepancy decreases when overshoot is included. Small changes in metallicity and amount of overshoot must be tested before turning to a more sophisticated treatment of the very inner convective regions. 


\section{Acknowledgements}

We gratefully thank J. Christensen-Dalsgaard for providing his code and helpful discussions, and W. Dziembowski for fruitful comments during this conference.

\section{References}

Christensen-Dalsgaard, J: 1982, Monthly Notices of the RAS 199, 735.

Christensen-Dalsgaard, J. and Gough D. O.: 1982, Monthly Notices of the RAS 198, 141. Dziembowski, W. A. and Goode, P. R.: 1992, preprint.

Dziembowski, W. A. and Krolikowska, M.: 1990, Acta Astron. 40, 19.

Dziembowski, W. A. and Pamyatnykh, A. A.: 1991, Astronomy and Astrophysics 248, L11.

Lee, U.: 1985, Publications of the ASJ, 37, 279.

Maeder, A. and Meynet, G.: 1989, Astronomy and Astrophysics 210, 155.

Michel, E. et al.: 1992a, Astronomy and Astrophysics, in press.

Michel, E., Goupil, M. J., Lebreton, Y., and Baglin, A.: 1992b, Astronomy and Astrophysics, in prep.

Rogers, F. J. and Iglesias, C. A.: 1991, preprint.

Saio, H.: 1981, Astrophysical Journal 244, 299. 